\title{
Cognition in psychiatry: population health, clinical, and research implications
}

\author{
Roger S. McIntyre (iD) *
}

Brain and Cognition Discovery, Mood Disorders Psychopharmacology Unit, University Health Network, University of Toronto, Toronto, Ontario, Canada

Received 26 March 2018; Accepted 4 April 2018; First published online 6 November 2018

The global population is expanding, becoming more urbanized, and "middle-class-ized." Moreover, lifespans globally are increasing, largely due to public health interventions (eg, sanitation, hygiene), as well as preventative and risk-factor modification (eg, immunization). To some extent, the lengthier lifespan is due to advances in pharmacotherapies across disparate medical areas. Coincident with the foregoing population and clinical trends is an increasing proportion of global disability attributable to mental disorders. The high incidence and prevalence, as well as relatively early age of onset, low rates of recovery, and the absence of diseasemodifying and/or curative therapy, account for the overall morbidity and increased costs due to mental disorders.

It is disquieting that the projection of human disability due to mental disorders is expected to increase over the foreseeable future. ${ }^{1}$ Impairment of workplace function is the largest cost associated with mental disorders, and is largely mediated by abnormalities in cognitive function. The workplace of the past required workers to be in possession of skill sets that were simple and manual, as many work environments were routine. The workplace of today and the future requires skill sets that are complex, cognitive, and non-routine. ${ }^{2}$ This change is a consequence of the digital revolution, which has demanded the availability of brain capital for the current "brain" or "cognitive economy." In keeping with this view, syndromes, disorders, and diseases that affect the central nervous system (CNS) represent the single greatest threat to brain capital.

Cognitive functions are variably defined and include "atoms" of executive function, attention/concentration, learning/memory, and processing speed, which are discrete functions that are "covalently bonded." Surreal

* Address for correspondence: Roger McIntyre, University Health Network - Brain and Cognition Discovery Foundation, 399 Bathurst Street, MP 9-325 Toronto, ON M5G 2C4, Canada.

(Email: Roger.McIntyre@uhn.ca) advances in cognitive neuroscience, fueled in part by available technology, have provided the opportunity to comprehensively parse the neurobiological substrates that subserve cognitive function. Rather than the anachronistic disease model, which implicates disturbance in monoaminergic function as a central and pervasive abnormality across mental disorders, the prevailing model today implicates alterations in reciprocity and functional connectivity within circuits, nodes, and networks (CNN).

A parallel body of literature, so called "mediational studies," has provided ample evidence that psychopathology differentially affects psychosocial and workplace function. It is well recognized that the phenomenology of mental disorders is overtly heterogeneous. Less appreciated is the covert heterogeneity of mental disorders referring to the complex inter-relationship between CNN and their associated phenomenological dimensions/ domains. For example, it is recognized that in adults with mood disorders, deficits in cognitive function account for more variability in psychosocial and workplace function than other domains of psychopathology (eg, mood disturbances). ${ }^{3}$ A lack of zone of delimitation between dimensions and domains of DSM-5-based psychopathology is a reminder of multi-linearity across domains of psychopathology including, but not limited to cognition. For example, it is expected that improvements in measures of motivation and reward would be associated with improvements in general cognitive processing. Notwithstanding, improvements in general cognitive processing pursue a separate and independent response to treatment and contribution to human morbidity.

This issue of CNS Spectrums is dedicated to the topic of cognition in mental disorders. We have invited leading experts globally who have contributed to the literature with independent thinking and have made substantial impact in this ecosystem. The series of articles attempts to be cohesive as well as comprehensive, wherein we attempt to strike the difficult balance between 
granularity and perspective. Articles in this dedicated issue cover the following: glossary for cognition; current knowledge as it relates to measurement of cognitive function; a synthesis of neuroscience regarding mechanistic substrates and novel heuristic models; and an up-todate summary of existing treatments and possibly prevention strategies for cognitive function. Novel strategies for cognitive function are covered, including but not limited to pharmacologic, psychological, and behavioral. We have also prepared an expert consensus on the assessment and screening of cognitive functions in individuals presenting for clinical care, agnostic of the disease state. The impetus to create the expert consensus was provided by general recognition of the ubiquity of, hazards posed by, and clinical relevance of cognitive functions in routine clinical practice.

Tacit to this special issue is a pivot in psychiatry toward dimensions/domains-psychopathology. Akin to dyslipidemia, wherein fractionating cholesterol moieties is relevant to risk stratification and treatment selection, increasingly in clinical and research-based psychiatry it is recognized that there is relevance to "fractionating" psychopathology (ie, all symptoms are not created equal). Admittedly, cognition is under-represented with interventional opportunities and/or FDA-approved interventions. Nonetheless, a wide assortment of primary and secondary prevention strategies is available, which should be prioritized in discussions between healthcare providers and patients. It is ruefully stated that psychiatric pharmacology has been disappointing with respect to reliably and robustly improving psychosocial and workplace functioning, as well as improving patient-centric outcomes. It is a viable and testable hypothesis that targeted therapies for cognitive functions across mental disorders will preserve and enhance human capital so that our patients can participate meaningfully in the public square and pursue the "gigs" that are most meaningful to them.

\section{Disclosures}

Dr. McIntyre reports grants from Allergan, grants from AstraZeneca, grants from Bristol-Myers, grants from Janssen-Ortho, grants from Lundbeck, grants from Otsuka, grants from Purdue, grants from Pfizer, grants from Shire, grants from Sunovion, grants from Neurocrine, and grants from Takeda, outside the submitted work.

\section{REFERENCES:}

1. Insel TR, Schoenbaum M, Wang PS, National Institute of Mental Health (NIMH). Components of the economic burden of serious mental illness in the US. US Neurology. 2009;5(1):10.

2. McIntyre RS, Lee Y. Cognition in major depressive disorder: a "systemically important functional index" (SIFI). Curr Opin Psychiatry. 2016;29(1):48-55.

3. McIntyre RS, Soczynska JZ, Woldeyohannes HO, et al. The impact of cognitive impairment on perceived workforce performance: results from the International Mood Disorders Collaborative Project. Compr Psychiatry. 2015;56:279-282. 